\title{
Optimizing carbon efficiency of jet fuel range alkanes from cellulose co- fed with polyethylene via catalytically combined processes
}

\author{
Xuesong Zhang, Hanwu Lei*, Lei Zhu, Xiaolu Zhu, Moriko Qian, Gayatri Yadavalli, Di Yan, Joan Wu, \\ Shulin Chen \\ Department of Biological Systems Engineering, Washington State University, Richland, WA \\ 99354-1671, USA.
}

*Corresponding author, Phone: 509-372-7628, Fax: 509-372-7690,E-mail: hlei@wsu.edu

\begin{abstract}
Enhanced carbon yields of renewable alkanes for jet fuels were obtained through the catalytic microwave-induced co-pyrolysis and hydrogenation process. The well-promoted ZSM-5 catalyst had high selectivity toward $\mathrm{C}_{8}-\mathrm{C}_{16}$ aromatic hydrocarbons. The raw organics with improved carbon yield ( 44\%) were more principally lumped in the jet fuel range at the catalytic temperature of $375{ }^{\circ} \mathrm{C}$ with the LDPE to cellulose (representing waste plastics to lignocellulose) mass ratio of 0.75 . It was also observed that the four species of raw organics from the catalytic microwave co-pyrolysis were almost completely converted into saturated hydrocarbons; the hydrogenation process was conducted in the $n$-heptane medium by using home-made Raney Ni catalyst under a low-severity condition. The overall carbon yield (with regards to co-reactants of cellulose and LDPE) of hydrogenated organics that mostly match jet fuels was sustainably enhanced to above $39 \%$. Meanwhile, $\sim 90 \%$ selectivity toward jet fuel range alkanes was attained.
\end{abstract}

KEYWORDS: Bio-jet fuels; co-feed catalytic microwave pyrolysis; hydrogenation; cellulose; low-density polyethylene 


\section{Introduction}

Municipal solid waste (MSW) has been found as one of the major challenges in the face of the modern world (Fang et al., 2016); in particular, the sustainable disposal management of MSW was intensively appealed in the industrialized and developing countries (Lemoine et al., 2013). MSW is comprised of various categories of materials. Among it, biomass co-existing with plastics make up a majority of the composition (Cheng \& Hu, 2010). Yet, the conventional methods for the disposal of MSW takes away valuable land and causes numerous environmental issues (Cheng \& $\mathrm{Hu}, 2010)$. More attentions are paid to energy-efficient, eco-friendly and economically sound technologies for the disposal of MSW. Because discarded MSW represents a tremendous energy source; MSW-to-energy technologies not only mitigates negative impacts on the environment, but also provides sustainable energy. Catalytic fast pyrolysis (CFP) is recognized as the most prevailing and promising pathway in a single process (Cheng \& Huber, 2012; Li et al., 2015; Li et al., 2013). Zeolite-based catalysts (e.g. ZSM-5) have been proved as the most efficient catalysts to produce important petrochemicals (Dorado et al., 2014; Li et al., 2013; Zhang et al., 2014).

However, even in the presence of ZSM-5 catalyst, catalytic pyrolysis of lignocellulose alone only generated low carbon yields $(10-30 \%)$ of aromatic hydrocarbons; large amounts of solid residues (carbon yields usually above 30\%) were also achieved in the process (Li et al., 2015; Liu et al., 2014; Yao et al., 2015). It is plagued by the high yield of coke deposited on the catalyst. That is because the coke could rapidly deactivate the catalyst and further reduce the 
lifetime, triggering the catalytic process to be impractical. These aforementioned phenomenon is principally due to the intrinsically oxygen rich nature and hydrogen deficiency of biomass (Liu et al., 2014; Xue et al., 2016; Yao et al., 2015). It was found that the hydrogen to carbon effective $\left(\mathrm{H} / \mathrm{C}_{\text {eff }}\right)$ ratio plays a vital role in the efficiency for converting biomass to biofuel and coke formation (Cheng \& Huber, 2012; Dorado et al., 2015; Zhang et al., 2015a). Thus the hydrogendeficient $\left(\mathrm{H} / \mathrm{C}_{\text {eff }}\right.$ usually less than 0.3$)$ biomass produce low yields of petrochemicals, but large amounts of coke when they were converted over zeolite-based catalysts (Li et al., 2013; Zhang et al., 2014).

To improve the carbon efficiency of aromatic production and reduce the formation of coke, the incorporation of hydrogen-enriched co-reactant in the catalytic pyrolysis of biomass could mitigate these problems. It is mostly likely that co-fed hydrogen-rich feedstock in the catalytic pyrolysis of biomass could change reaction mechanism of oxygen removal from biomass by substituting decarbonylation and decarboxylation with deoxygenation (Dorado et al., 2015; Green et al., 2016; Li et al., 2015). It is widely known that waste plastics represent a cheaper and abundant hydrogen source (Li et al., 2014b; Li et al., 2013; Xue et al., 2016; Yao et al., 2015; Zhang et al., 2014), which were utilized to improve carbon yield of aromatic production and lower the coke formation in the co-feed catalytic pyrolysis. For example, polyethylene accounts for up to $40 \%$ of gross waste plastics in MSW (Miskolczi et al., 2004). Accordingly, the cofeeding of biomass with waste plastics from MSW in catalytic pyrolysis is beneficial for the environment and energy recapture. 
On the other hand, the co-feeding of biomass with plastics in catalytic pyrolysis commonly gave rise to mono-ring aromatic hydrocarbons with low carbon number $\left(\mathrm{C}_{6}-\mathrm{C}_{8}\right)$ (Green et al., 2016; Li et al., 2014b; Xue et al., 2016; Zhang et al., 2015a). These hydrocarbons with low carbon numbers cannot suit current jet fuels within $\mathrm{C}_{8}-\mathrm{C}_{16}$ hydrocarbons. Linear-chain and branchedchain alkanes predominantly make up $\mathrm{C}_{8}-\mathrm{C}_{16}$ hydrocarbons in the current jet fuels (Bi et al., 2015; Huber et al., 2006). By contrast, cycloalkanes are compact molecules within robust ring strain and can be burned cleanly with high heats of combustion (Li et al., 2014a; Zhang et al., 2015f). As expected, jet fuel range cycloalkanes should be synthesized and added into commercial jet fuels (e.g. Jet A and JP-8). It was noteworthy that aromatic hydrocarbons are prone to be transformed into cycloalkanes by hydro-cycloaddition reactions under a mild reaction condition (Zhang et al., 2015d; Zhang et al., 2015e). To pursue renewable cycloalkanes, highly desirable aromatic hydrocarbon $\left(\mathrm{C}_{8}-\mathrm{C}_{16}\right)$ as the precursors should be synthesized in cofeed catalytic pyrolysis, rather than mono-ring aromatic hydrocarbons with low carbon numbers.

Parent ZSM-5 zeolite used for aromatic production with low carbon number are not suitable for the production of jet fuel range aromatics in the catalytic pyrolysis. Nonetheless, moderate treatments were employed to tailor zeolite properties for modifications of porosity and acidity, which is essential for improving product selectivity of $\mathrm{C}_{8}-\mathrm{C}_{16}$ aromatic hydrocarbons in our previous study (Zhang et al., 2015b). The mild hydrothermal and calcined conditions favored the generation of mesopores for the diffusion of double-ring aromatics, and catalytic sites for specific efficiency. In this regard, we have developed a tandem process to produce renewable cycloalkane for jet fuels derived from lignocellulosic biomass individually, albeit achieving a low carbon yield (24.68\%) (Zhang et al., 2015e). It is manifested that microwave-assisted 
pyrolysis technology is one of the most promising methods for enhancing and accelerating chemical reactions due to effective heat transfer through microwave irradiation (Luque et al., 2012). In comparison with conventional pyrolysis, microwave-assisted pyrolysis encloses the potentials of fast and selective heating, easy control of reaction conditions, low reaction temperatures and energy requirements (Lidstrom et al., 2001). In the hydrotreating process, Raney-type $\mathrm{Ni}$ is widely employed as a versatile catalyst for reductive conversions of organic compounds (Gross et al., 2001). It was discerned that the catalytic activities of home-made Raney Ni catalyst used was superior to as-purchased Raney Ni catalysts (Zhang et al., 2015e). We observed that aromatics from catalytic microwave pyrolysis were almost completely converted into high-density cycloalkanes in the $n$-heptane medium under a low-severity condition.

This present work aims to unravel the key interactions between cellulose and low-density polyethylene (LDPE), which act as the model compounds of lignocellulosic biomass and waste plastics in MSW. To this end, cellulose and LDPE were first co-pyrolyzed using a tandem microwave-induced system coupled with a downstream catalysis system. For converting aromatics into renewable cycloalkanes for jet fuels, several species of liquid organics extracted by the optimum solvent ( $n$-heptane) was transformed into saturated hydrocarbons by using home-made Raney Ni catalyst in the hydrogenation process. The influence of organic species in the overall carbon yield and product distribution of saturated hydrocarbon in the jet fuel range was also evaluated.

\section{Materials and methods}




\subsection{Materials}

Cellulose (CAS number 9004-34-6), in the form of microcrystalline powders and particle sizes is averaged at $50 \mu \mathrm{m}$, was purchased from Sigma-Aldrich Corporation (St. Louis, MO, USA). Low-density polyethylene (LDPE) (CAS number 9002-88-4) in the form of pellets was purchased from Sigma-Aldrich Corporation (St. Louis, MO, USA). The density and melting point of LDPE are $0.925 \mathrm{~g} / \mathrm{cm}^{3}$ at ambient temperature and $116{ }^{\circ} \mathrm{C}$, respectively. The elemental composition of cellulose and LDPE is described in Table 1. Parent ZSM-5 $\left(\mathrm{SiO}_{2} / \mathrm{Al}_{2} \mathrm{O}_{3} \mathrm{Mole}\right.$ Ratio: 50) was purchased from Zeolyst International, USA. Nickel-Aluminum alloy powder in a non-activated type was used as purchased from Alfa Aesar (Ward Hill, MA, USA).

\subsection{Catalyst preparation}

The activity of parent ZSM-5 was improved by both hydrothermal and calcined treatments. Under the gentle stirring, parent ZSM-5 powder was added into deionized water (mass ratio=1) at $60{ }^{\circ} \mathrm{C}$. After addition, the mixture was kept on stirring for $2 \mathrm{~h}$ under this condition. The slurry was then dried at $105{ }^{\circ} \mathrm{C}$ till constant weight. The sequential process was the catalyst calcination: hydrothermally treated ZSM-5 was calcined at $550^{\circ} \mathrm{C}$ for $5 \mathrm{~h}$ in a muffle furnace. The catalysts were pelletized and sieved to $20-40$ mesh.

Home-made Raney Ni catalyst was developed using a $20 \mathrm{wt} \% \mathrm{NaOH}$ aqueous solution to remove $\mathrm{Al}$ in the following procedure. $10 \mathrm{~g}$ of the above $\mathrm{Ni}-\mathrm{Al}$ was slowly added into $100 \mathrm{~mL}$ $\mathrm{NaOH}$ aqueous solution under gentle stirring. After addition, the slurry was kept on stirring at $80{ }^{\circ} \mathrm{C}$ for $1 \mathrm{~h}$. The excess of sodium hydroxide was finally washed with distilled water until 
nearly neutral pH was reached. The obtained Raney Ni catalyst was stored in water. Raney Ni is notorious for its pyrophoricity, and it may ignite spontaneously when dried in air. The Raney Ni catalyst was thus dried at $60{ }^{\circ} \mathrm{C}$ till constant weight in the atmosphere of nitrogen to avoid contact with air, prior to the subsequent catalytic test. The textural properties of the wellpromoted ZSM-5 and home-made Raney Ni catalysts are listed in Table 2.

\subsection{Co-feed catalytic microwave-induced pyrolysis of cellulose and LDPE}

Fig. S1 presents the schematic diagram of the microwave-assisted pyrolysis system coupled with the catalysis system. Detailed experimental setting was described in our previous studies (Zhang et al., 2015c). Cellulose powder was first air dried at $105^{\circ} \mathrm{C}$ for $24 \mathrm{~h}$ to remove the physically bound moisture, prior to conducting the experiments. Co-feeding of cellulose with LDPE were placed in a $500 \mathrm{~mL}$ quartz flask which was placed inside a Sineo MAS-II batch microwave oven (Shanghai, China) by a constant microwave power setting $(700 \mathrm{~W}) .0 .05 \mathrm{~g}$ of activated carbon powder was used as the absorber for the microwave-assisted pyrolysis. All reactions of microwave pyrolysis were conducted at the temperature of $480{ }^{\circ} \mathrm{C}$ for $10 \mathrm{~min}$ to assure the sufficient interactions between cellulose and LDPE.

\subsection{Hydrogenation of liquid organics derived from co-feed catalytic microwave pyrolysis}

According to the solvents influence in the hydro-cycloaddition of a model compound (naphthalene) in our previous study (Zhang et al., 2015e), the combined bio-oils evolved from co-feed catalytic microwave pyrolysis were extracted by the optimal solvent ( $n$-heptane). To produce renewable cycloalkanes for jet fuels, a closed reaction system with a stirred stainless 
batch reactor of the 4592 micro stirred reactor (with a $50 \mathrm{~mL}$ vessel) and a 4848 reactor controller from Parr Instrument Company (Moline, IL, USA) was used (Fig. S2). The mixture of organics and the $n$-heptane medium was loaded into the reactor together with 20 wt $\%$ homemade Raney Ni catalyst (in terms of the reactants). Then the reactor was sealed and vented for five times with hydrogen to get rid of the air present in the vessel. Hydrogen was subsequently adjusted to reach the set pressure (500 psi). The automatic controller was employed to control the temperature and the revolution of stirrer $(300 \mathrm{rpm})$. The pressure inside the reactor was recorded and the reactions proceeded at $200{ }^{\circ} \mathrm{C}$ for $2 \mathrm{~h}$. After the experiment finished, stirring was stopped and the reactor was rapidly cooled to ambient temperature. Then, the gas was collected for analysis and the reactor was depressurized. Consequently the liquid product was filtered to remove catalyst particles.

\subsection{Analytical techniques}

Elemental analysis (C, H and N) of feedstock, liquid samples, char, and coke deposited on spent catalysts was conducted using a 2400 Series II CHN/O Elemental Analyzer (PerkinElmer, USA).

The chemical composition of the liquid products was characterized by Agilent 7890A GC-MS (GC-MS; GC, Agilent 7890A; MS, Agilent 5975C) with a DB-5 capillary column. The carbon moles of main compounds in liquid products were quantified by the normalization method with standard samples. The GC was first programmed to heat to $45^{\circ} \mathrm{C}$ for 3 min followed by heating to $300^{\circ} \mathrm{C}$ at a rate of $10^{\circ} \mathrm{C} / \mathrm{min}$. The injection sample size was $1 \mu \mathrm{L}$. The flow rate of the carrier gas (helium) was $0.6 \mathrm{~mL} / \mathrm{min}$. The ion source temperature was $230{ }^{\circ} \mathrm{C}$ for the mass selective detector. Compounds were identified by comparing the spectral data with that in the NIST Mass 
Spectral library. The area percent of changed concentrations of model compounds obtained from GC/MS results was utilized to predict product concentration in bio-oils. All the measurements were triplicated to assure reproducibility.

The moisture content in the bio-oils was determined by a Karl Fischer (KF) compact titrator (V20 Compact Volumetric KF Titrator, Mettler-Toledo).

The gaseous product was collected in a 1L Tedlar gas bag and then offline analyzed by an INFICON 3000 Micro-GC (INFICON Inc., Santa Clara, CA, USA) system with a thermal conductivity detector (TCD). A standard gas mixture consisting of $\mathrm{H}_{2}, \mathrm{~N}_{2}, \mathrm{CH}_{4}, \mathrm{CO}, \mathrm{CO}_{2}, \mathrm{C}_{2} \mathrm{H}_{4}$, $\mathrm{C}_{2} \mathrm{H}_{6}$, and $\mathrm{C}_{3} \mathrm{H}_{6}$ was used to calibrate the yield of non-condensable gas. Alkanes and olefins $\left(>\mathrm{C}_{4}\right)$ in gas samples were either not detected or negligible in this research. All the measurements were triplicated to assure reproducibility.

\subsection{Experimental methods and data evaluation}

A central composite experimental design (CCD) was employed to optimize the process conditions and product yields from co-feeding of cellulose and LDPE in catalytic microwave pyrolysis (Table 3$)$. The catalytic temperature $\left(\mathrm{X}_{1},{ }^{\circ} \mathrm{C}\right)$ and $\mathrm{LDPE}$ to cellulose ratio $\left(\mathrm{X}_{2}\right)$ were chosen as independent variables. The loading of cellulose was $20 \mathrm{~g}$ for each run, while the catalyst to co-reactants ratio was kept constantly at 0.2 in the co-feed catalytic microwave pyrolysis. In these experiments based on CCD, the mass of LDPE pellets varied from 8 to $22 \mathrm{~g}$, while packed bed catalytic temperature ranged from 269 to $481{ }^{\circ} \mathrm{C}$. Four additional experiments 
were conducted as the controls (Entry $14-17$, Table 3).

The coke mass was determined by the difference before and after catalytic co-pyrolysis. Because of ex-situ catalysis, char was left in the quartz flask inside microwave reactor; while the coke was formed on the well-promoted ZSM-5 catalyst in the packed-bed catalysis reactor. The weight of non-condensable gas was calculated using the following equation.

Weight of gas = initial reactants mass - liquid mass - char mass - coke mass

Overall carbon yields of the liquid, gas, and solid products and carbon selectivity of a specific product were calculated based on the following equations.

$$
\begin{aligned}
& \text { Carbon yield }=\frac{\text { moles of carbon in a product }}{\text { moles of carbon fed in }} \times 100 \% \\
& \text { Carbon seletivity }=\frac{\text { moles of carbon in a product }}{\text { moles of carbon in identified products }} \times 100 \%
\end{aligned}
$$

\section{Results and discussion}

\subsection{Product yield distributions from co-feed catalytic microwave pyrolysis}

The product yield distribution from co-feeding of cellulose with LDPE in the catalytic microwave pyrolysis are listed on the basis of catalytic temperature and LDPE to cellulose mass ratio in Table 3. The mass yields of bio-oil and non-condensable gas were strikingly affected by the two variables in the range from 33.54 to $51.45 \mathrm{wt} \%$ versus 37.13 to $48.55 \mathrm{wt} \%$, respectively. The optimal condition for maximizing bio-oil yield was presented to be at the catalytic 
temperature of $375^{\circ} \mathrm{C}$ with the LDPE to cellulose ratio of 1.45 ; while the maximum gas yield was predicted at $375{ }^{\circ} \mathrm{C}$ with the absence of LDPE. In general, the water content in the bio-oil varied from 9.67 to $29.74 \mathrm{wt} \%$ depending on the reaction conditions. Without the application of LDPE in the catalytic microwave pyrolysis, the water content could attain $30 \mathrm{wt} \%$. However, the co-feeding of LDPE in the catalytic microwave pyrolysis of cellulose reduced the water content down to $10 \mathrm{wt} \%$ in the bio-oil. As discerned, olefins from thermal decomposition of LDPE could react with cellulose-derived furans to form aromatic hydrocarbons through DielsAlder reactions followed by dehydration at Brönsted acid sites (Williams et al., 2012). As a result, if the extent of dehydration reaction was enhanced in the co-feed catalytic microwave pyrolysis; the increasing trend of water content in the bio-oil was observed. Furthermore, the production of aromatic hydrocarbon from catalytic cracking of olefins and subsequent cyclization was also influenced by the two independent variables (Zhang et al., 2015c).

Solid carbonaceous residue (char and coke) from thermal degradation and catalytic reforming can be distinguished because of the ex-situ catalysis. It can be seen that catalytic temperature and LDPE to cellulose ratio affected mass yields of both char and coke. The char yield was in the range from $7.03 \mathrm{wt} \%$ at the highest LDPE to cellulose ratio of 1.45 to $16.09 \mathrm{wt} \%$ without the usage of LDPE. The char residue from the co-feed catalytic microwave pyrolysis was lower than catalytic microwave pyrolysis of cellulose alone. These results indicate that co-feeding of cellulose with LDPE in the catalytic microwave pyrolysis was had a pronounced impact on the char formation. It is mainly due to the fact that with the presence of hydrogen originating from the thermal decomposition of LDPE, the re-polymerization reactions of oxygenates evolved from cellulose were partially inhibited ( $\mathrm{Li}$ et al., 2014b). Therefore the char formation was strongly 
reduced when cellulose was co-pyrolyzed with LDPE.

Given the coke deposited on the catalyst resulting in the loss of the active sites and micropores blockage (Zhang et al., 2015b), the co-feed catalytic microwave pyrolysis is necessitated to aid in coke reduction and the extension of catalyst lifetime. The coke deposition yield with regard to catalytic temperature and LDPE to cellulose ratio is also depicted in Table 3, ranging from 0.57 to $1.82 \mathrm{wt} \%$. As expected, the coke deposition was impacted by the co-feeding of LDPE, which abated the formation of coke precursors. It was found that the highest yield of coke was obtained with the absence of LDPE. Formation and deposition of coke was considerably attenuated as LDPE was co-fed. It has been suggested that in the presence of ZSM-5 catalyst, cellulosederived furans could react with LDPE-derived olefins via Diels-Alder reactions followed by dehydration reactions, thereby reducing the coke formation from polymerization of furans (Cheng \& Huber, 2012; Li et al., 2013). Furthermore, plastics-derived hydrocarbons could provide hydrogen for cellulose-derived oxygenates, mitigating the coke formation from hydrogen-deficient oxygenates in the zeolite-catalyzed conversions (Li et al., 2014b). For ANOVA analysis of the experiment based on CCD, the $P$-value of the four models (the bio-oil, gas, char, and coke yield) are all less than 0.05, implying the models are significant to present the relationships between product yields and the two independent variables as shown in Fig. S3 - S6. The coefficient of determination $\left(R^{2}\right)$ for the four models are all larger than 0.96 , evidencing that these models fairly represent the relationships with respect to the independent variables. Consequently, these four models can be used to predict the maximum product yields in accordance to the two variables. 


\subsection{The effect of catalytic temperature on the co-feed catalytic microwave pyrolysis}

As reported in our previous studies, catalyst temperature had a pronounced effect on product distribution by using ZSM-5 as the catalyst (Zhang et al., 2015b). In this respect, catalytic temperature was chosen as the variable to investigate the product distribution and even evaluate the quality of liquid organics. Representative results of carbon yields regarding product distribution from co-feed catalytic microwave pyrolysis were at an array of catalytic temperature between 250 and $500{ }^{\circ} \mathrm{C}$ at the same plastics to biomass ratio (0.75) as shown in Fig. 1. The carbon yield of char did not change alongside the variation of catalytic temperature, since the packed-bed catalysis reactor was located downstream from the microwave pyrolysis reactor. Notably, increasing the catalyst temperature during co-feed catalytic microwave pyrolysis decreased the carbon yield of coke. Since direct decomposition of plastics produced a large amount of long-chained waxes (Xue et al., 2016), these waxes were readily adsorbed on the catalytic surface at the low catalytic temperature, generating the waxy coke that enveloped the whole catalyst. Likewise, the polymerization of aromatics with oxygenates to form coke also took place at the low catalytic temperature. When the catalytic temperature was elevated, catalytic cracking of waxes absorbed on the catalyst was facilitated, contributing to lighter compounds that were more easily accessible to the pores. Thus the carbon yield of coke decreased at the expense of large molecules cracking as the catalytic temperature was enhanced.

Because the thermal decomposition of plastics is an endothermic reaction, the yield of plasticsderived olefins should go up as the catalytic temperature increases at the tested region (Zhang et 
al., 2014). The increasing trend from degradation of plastics could strengthen the Diels-Alder reaction, contributing to the increase of liquid aromatics (Zhang et al., 2015a). Nonetheless, an excessively high catalytic temperature resulted in reverse Diels-Alder reactions to produce light olefins instead of liquid aromatics (Cheng \& Huber, 2012; Li et al., 2014b). It was also manifested that increasing catalytic temperature favored the catalytic cracking of oxygenates into non-condensable gas, preventing the formation of liquid aromatics (Cheng \& Huber, 2012). Consequently, the carbon yield of liquid organics gradually decreased owing to the elevated catalytic temperature. By contrast, the carbon yield of gas monotonically enhanced at the expense of liquid organics formation as the catalytic temperature went up to $500{ }^{\circ} \mathrm{C}$.

The detailed carbon yields and selectivity corresponding to catalytic temperature can be more clearly discerned in Table 4. As for the liquid organics from co-feed catalytic microwave pyrolysis at $250{ }^{\circ} \mathrm{C}$, large amounts of aliphatic olefins and cellulose-derived oxygenates were produced in the liquid organics. This result indicates that such low catalytic temperature could not favor the zeolite-catalyzed reactions to form aromatics. As the catalytic temperature went up to $375^{\circ} \mathrm{C}$, there was a minor amount of aliphatic hydrocarbons detected in the liquid organics. Besides, aromatic oxygenates (such as phenol) existing in the liquid organics was much lower than those from catalytic microwave pyrolysis of biomass individually under the same conditions. It is likely that LDPE-derived olefins participated in the Diels-Alder reaction with cellulosederived furans followed by dehydration reaction or directly aromatize to achieve enhanced aromatic hydrocarbons. As listed in Table 4, aromatic hydrocarbons were predominantly composed of toluene, xylenes, trimethylbenzenes, indane, indene, naphthalene, and their derivatives. The carbon selectivity toward ethylbenzene and xylenes ranged from 6.76 to $8.02 \%$ 
and 18.95 to $26.92 \%$, respectively; whilst the carbon selectivity toward trimethylbenzene declined from 15.21 to $13.01 \%$ as catalytic temperature increased from 250 to $500{ }^{\circ} \mathrm{C}$. These outcomes suggest that the dealkylation reaction of aromatics was also promoted at the elevated catalytic temperature, leading to increase of simple aromatic hydrocarbons (Xue et al., 2016; Zhang et al., 2014). Comparing with the amount of oxygenates (e.g. phenol) achieved at low catalytic temperature, only a trace amount of oxygenates was obtained at the catalytic temperature of $500{ }^{\circ} \mathrm{C}$. It could be inferred from these results that under such condition, hydrogen transfer reactions could promote the cracking of the side chains substituted on phenyl on the catalyst surface to form aromatic hydrocarbons (Mullen \& Boateng, 2010). To make the precursors lumped in the jet fuel range, we observed that the aromatic hydrocarbons obtained at catalytic temperature of $375^{\circ} \mathrm{C}$ were more desirable.

Non-condensable gas was another major co-product from co-feeding of cellulose with LDPE in the catalytic microwave pyrolysis. The composition of gaseous fraction with respect to catalytic temperature is also depicted in Table 4. Because of the co-feed catalytic pyrolysis, oxygen content was expelled in the form of water from furans by reacting with olefins through DielsAlder reactions followed by dehydration reaction, rather than decarbonylation and decarboxylation reactions which produce $\mathrm{CO}$ and $\mathrm{CO}_{2}$ (Cheng \& Huber, 2012; Williams et al., 2012). Thus the carbon yields of both $\mathrm{CO}$ and $\mathrm{CO}_{2}$ were very low. However, it was observed that the carbon yields of both $\mathrm{CO}$ and $\mathrm{CO}_{2}$ increased as the catalytic temperature go up from 375 to $500{ }^{\circ} \mathrm{C}$. It is reaffirmed that the elevated catalytic temperature had a negative impact on the Diels-Alder reactions (Xue et al., 2016), rejecting oxygen content from oxygenates by decarbonylation and decarboxylation reactions. It is worth noting that the carbon yield of 
ethylene gradually increased as the catalytic temperature went up to $500{ }^{\circ} \mathrm{C}$. It is inferred that increasing catalytic temperature accelerated catalytic cracking of waxes (long-chain hydrocarbons) into light olefins. In addition, elevated catalytic temperature also prevented light olefins from undergoing the aromatization reaction to form aromatic hydrocarbons ( $\mathrm{Li}$ et al., 2015; Zhang et al., 2014).

\subsection{The effect of LDPE to cellulose ratio on the co-feed catalytic microwave pyrolysis}

It was ensured that the optimal condition to obtain the lumps of aromatics hydrocarbons for jet fuels was set at $375{ }^{\circ} \mathrm{C}$. Since cellulosic biomass is more abundant and cheaper than waste plastics, it is desired to enhance the mass ratio of cellulosic biomass in the co-reactants if this cannot decrease the carbon yield of liquids substantially. To evaluate how the effect of LDPE proportion affected the product distribution in the co-feed catalytic microwave pyrolysis, the overall carbon yield of product distribution at $375{ }^{\circ} \mathrm{C}$ as a function of LDPE to cellulose ratio is shown in Fig. 2. The carbon yield of liquid organics increased nonlinearly with the increase of LDPE to cellulose ratio. In comparison with the run conducted in the absence of LDPE, the carbon yield of liquid organics rapidly increased as the ratio went up to 0.75 . This increasing trend indicates that there was a positive synergy between the co-reactants. This trend can be easily rationalized because the degradation of LDPE produced large amounts of olefins with high $\mathrm{H} / \mathrm{C}_{\text {eff }}$ ratio, reacting with cellulose-derived oxygenates by the Diels-Alder reaction to augment liquid organics yield (Zhang et al., 2012). It is manifested that the yields of liquid organics from co-feed catalytic microwave pyrolysis were much higher than the optimal result (above 30\%) from catalytic microwave pyrolysis of LDPE alone (Zhang et al., 2015c). Nevertheless, as the 
LDPE to cellulose ratio increased from 0.75 to 1.45 , the carbon yield of liquid organics slightly went up. It has been suggested that when LDPE was fed in excess relative to cellulose, the conversion of excessive olefins into aromatic hydrocarbons required multiple reaction steps (e.g. oligomerization, cyclization, and aromatization reactions ) and was therefore less efficient (Li et al., 2014b). Consequently, the carbon yield of liquid organics was not significantly improved as the LDPE to cellulose ratio increased from 0.75 to 1.45 .

The carbon yields of char and coke gradually decreased with the increase of LDPE to cellulose ratio as shown in Fig. 2. It was found that both the carbon yields of char and coke dramatically declined as the LDPE to cellulose ratio went up to 0.75 ; while they show slight increasing tendencies as the ratio started 0.75 to 1.45 . It is reaffirmed that there was a synergistic effect between the co-reactants in the ratio range from 0 to 0.75 ; whereas excessive LDPE could not further enhance the synergy. Hence, there exists an optimal LDPE to cellulose ratio $(0.75)$ for the Diels-Alder reaction in co-feed catalytic microwave pyrolysis. The increasing carbon yield of gas with the increasing LDPE to cellulose ratio was attained. As outlined above, the Diels-Alder reaction could not be further enhanced at the high ratios, thus the light olefins from the catalytic cracking of LDPE assisted the carbon yield of non-condensable gas. The recyclability of wellpromoted ZSM-5 catalyst in the co-feed catalytic microwave pyrolysis was also estimated as sketched in Fig. S7. It was noticed that the carbon yield of liquid organics was dramatically affected by the catalyst recycle times, showing a slight decrease with the increase of recycle times. 
Table 5 lists how the carbon selectivity for the aromatic species changes with the addition of LDPE. In general, the carbon selectivity of main monocyclic aromatics first went up and then decreased as the LDPE to cellulose ratio increased; whist the carbon selectivity of main polycyclic aromatics gradually declined. For instance, the maximum carbon selectivity of aromatic hydrocarbons was xylenes, which increased from 20.24 to $23.33 \%$ and then declined to 21.47. Unlike the xylenes, the second abundant carbon selectivity toward aromatics was trimethylbenzene, showing a decreasing tendency from 17.45 to $13.12 \%$. On the other hand, the carbon selectivity toward polycyclic aromatics, such as naphthalene, experienced an appreciable drop as the LDPE to cellulose ratio increased. It is attributed to the fact LDPE-derived olefins competed with monocyclic aromatics for reacting with cellulose-derived oxygenates, which suppressed the polymerization reaction of monocyclic aromatics to form polycyclic aromatics (Li et al., 2014b). From the perspective of aromatic production with the carbon number in the jet fuel range, the run conducted at the LDPE to cellulose ratio of 0.75 was preferred in the co-feed catalytic microwave pyrolysis. Adding more LDPE would not considerably improve the carbon yield of liquid yield; yet, it would be a waste of LDPE.

The gaseous composition with respect to LDPE to cellulose ratio is also demonstrated in Table 5. The carbon yield of methane gradually decreased with the increase of LDPE to cellulose ratio; whereas, the carbon yield of both $\mathrm{CO}$ and $\mathrm{CO}_{2}$ decreased. It is mainly attributed to the DielsAlder reaction that was facilitated between cellulose and LDPE, instead of the decarbonylation and decarboxylation reactions. Unlike the $\mathrm{CO}$ and $\mathrm{CO}_{2}$, the carbon yield of ethylene was found to increase in the most pronounced way from 6.67 to $48.47 \%$, which in turn made the carbon yields of $\mathrm{CO}$ and $\mathrm{CO}_{2}$ decreased. These outcomes verifies that the increasing yield of ethylene 
could originate from catalytic cracking of waxes that was from the thermal degradation of LDEP. Besides, cellulose-derived oxygenates could promote the degradation of large molecules to light olefins in the co-feed catalytic pyrolysis (Zhang et al., 2014).

\subsection{Hydrogenation of liquid organics derived from co-feed catalytic microwave pyrolysis for jet} fuels

Considering the negative effect of water in the hydrogenation process (Zhang et al., 2015d), the small amount of water in the raw bio-oil from the co-feed catalytic microwave pyrolysis should be removed. Since $n$-heptane played an important role in the hydrogenation process (Zhang et al., 2015e), the raw bio-oil was thus separated and extracted by the optimum solvent ( $n$-heptane). The liquid organics and $n$-heptane in every samples were separated and weighed to measure the loss of organics in the extracting step. As the liquid organics produced by the feeding of cellulose with LDPE in the catalytic microwave pyrolysis (at the catalytic temperature of $375^{\circ} \mathrm{C}$ with the LDPE to cellulose ratio of 0.75 ) principally consisted of $\mathrm{C}_{8}-\mathrm{C}_{16}$ aromatics, the controllable adjustment of aromatic hydrocarbons with 8 - 16 carbon numbers are considered as precursors that should be converted into jet fuels. In addition, the run that obtained the maximum carbon yield of liquid organics (at the catalytic temperature of $375^{\circ} \mathrm{C}$ with the LDPE to cellulose ratio of 1.45) and the run that obtained the liquid organics within the lowest amount of oxygenates (at the catalytic temperature of $500{ }^{\circ} \mathrm{C}$ with the LDPE to cellulose ratio of 0.75 ) were hydrogenated for the optimization of jet fuels. Liquid organics acting as the control from the experiment that cellulose was subjected to catalytic microwave pyrolysis alone was hydrogenated as well. Furthermore, the elemental analysis corresponding to four species of raw 
organics was given in Table S1. As reported previously, naphthalene was completely transformed into saturated decalin in the $n$-heptane medium (Zhang et al., 2015e); thus the mass ratio of reactant to solvent was set at 1:7 for the following hydrogenation process.

The product distribution and the carbon selectivity toward main alkanes from the hydrogenation of diverse raw organics by using the home-made Raney $\mathrm{Ni}$ as the catalyst are elucidated in Table 6. Unreacted hydrogen (over 99 vol\%) was detected at the end of all reactions, suggesting that the reactions were not conducted under hydrogen starved conditions. The overall carbon yields of these hydrogenated organics (L-1, L-2, L-3, and L-4) with respect to the co-reactants of cellulose and LDPE were 21.91, 39.18, 34.71 and 40.08\%, respectively. These overall carbon yields of hydrogenated organics for jet fuels were much higher than that in our previous research (Zhang et al., 2015e). Among the hydrogenated organics, the result for the production of cycloalkanes from hydrogenated L-3 was superior to the others under the same condition. It was observed that more than $90 \%$ selectivity towards cycloalkanes was achieved from hydrogenated L-3. The high amounts of high-density cycloalkanes could be potentially used as high-density jet fuel (e.g., JP-10 and RJ-5). Yet it was found that the overall carbon yield of the hydrogenated organics was a little lower than those of both hydrogenated L-2 and L-4. For the production of aliphatic alkanes, hydrogenated L-4 obtained the maximum selectivity (31.45\%). From this perspective, the contents of hydrogenated L-4 were consistent with that in JP-5, containing $31.23 \%$ of aliphatic alkanes, $53.06 \%$ of cycloalkanes, and $15 \%$ of remaining aromatic hydrocarbons (Keil et al., 2003). The total amounts of hydro-aromatic hydrocarbons and aromatic hydrocarbons were less than $15 \%$, which meet the specifications of commercial jet fuels. A minor mount of other compounds were found in the all hydrogenated organics except 
hydrogenated L-1. As mentioned above, these were some oxygenates in the L-1 from catalytic microwave pyrolysis of cellulose alone. These oxygen content could not be removed by hydrogenation reaction under the low-severity condition; for example, phenol in L-1 was just convert into cyclohexanol via hydro-cycloaddition reaction. Less than 1 vol\% of small hydrocarbons (such as methane, ethane, and propane) were obtained, which suggests that there was almost no carbon loss by hydrocracking reaction in the hydrogenation system. In addition, the recyclability of the Raney $\mathrm{Ni}$ catalyst without regeneration was also determined in the hydrogenation process as shown in Fig S8. It was discerned that there was almost no any loss of catalytic activity in the second run, indicating that the resilience and durability of Raney Ni catalyst were perfect.

It was found that the maximum carbon selectivity toward main monocyclic alkanes were all from hydrogenated L-3, except trimethylcyclohexanes. Trimethylcyclohexanes derived from the hydro-cycloaddition of trimethylbenzenes had the highest carbon selectivity from hydrogenated L-1. That is possibly because the dealkylation reaction was favored at the high catalytic temperature $\left(500{ }^{\circ} \mathrm{C}\right)$. It is noticeable that the maximum carbon selectivity of main aliphatic alkanes was all achieved from hydrogenated L-4, which is reaffirmed that the high LDPE loading contributed to the high carbon yield of aliphatic hydrocarbons. Of the four raw organics for hydrogenation, the hydrogenated organics derived from L-2 produced the highest amounts of saturated alkanes matching jet fuels. With the consideration of both overall carbon yield and product distribution, the raw organics (L-2) is thus the optimal source to manufacture renewable jet fuels. 
3.6 Reaction pathway for the conversion regrading co-feeding of cellulose with LDPE into jet fuels

These observations are the key point to propose the reaction pathway for the conversion regarding co-feeding of cellulose with LDPE into jet fuels. The results of the hydrogenated organics for jet fuels were evidenced that the reaction pathway was more complicated than those mentioned previously (Dorado et al., 2014; Li et al., 2014b; Li et al., 2013). Based on the quantified products distribution in this study, related results from catalytic microwave-induce degradation of LDPE (Zhang et al., 2015c), and lignocellulosic biomass to jet fuel through combined catalytic conversions (Ren et al., 2012; Zhang et al., 2015b); the overall reactions network (including co-feed catalytic microwave pyrolysis and hydrogenation process) is proposed in Fig. 3. In the primary route regrading cellulose, cellulose underwent a series of dehydration, decarboxylation, and decarbonylation to form furan compounds (e.g. furfural) during thermal degradation (Cheng \& Huber, 2012; Zhang et al., 2015b). For the decomposition of LDPE by another route, Thermal degradation of LDPE took place through two mechanisms: random scission and chain-end scission (Artetxe et al., 2012). The two aforementioned mechanisms occurred simultaneously, giving rise to free radicals together with the long carbon chains (Serrano et al., 2005). Meanwhile, the radical fragments could also be transformed into straight chain dienes, alkenes and alkanes through hydrogen transfer reactions (Serrano et al., 2005). The hydrogen from the thermal degradation of LDPE could be provided for cellulosederived oxygenates that acted as the strong acceptor of hydrogen, suppressing the char formation. 
The long-chain hydrocarbons from the thermal degradation of LDPE could subsequently go through catalytic cracking in the presence of well-promoted ZSM-5 catalyst by two carbocationic mechanisms to form light olefins (Artetxe et al., 2012). These olefins could react with furans through the Diels-Alder reaction followed by the dehydration reaction to generate aromatic hydrocarbons. Meanwhile, these LDPE-derived olefins could individually subject to oligomerization, cyclization and aromatization reactions to form aromatic hydrocarbons (Xue et al., 2016). Likewise, these furans derived from cellulose could go through decarbonylation, aromatization, and oligomerization reactions inside the pores of well-promoted ZSM-5 catalyst to form aromatic hydrocarbons alone. As reported previously, the interactions through the hydrocarbon pool mechanism could exist in addition to Diels-Alder reaction (Li et al., 2013). Since the carbon yield of liquid organics significantly increased and the coke yield dramatically decreased as the LDPE was introduced, the Diels-Alder reaction was thus the dominant reaction pathway during the co-feed catalytic microwave pyrolysis in comparison to the hydrocarbon pool mechanism.

Furthermore, it was observed that the liquid organics resulting from the co-feed catalytic microwave pyrolysis mostly produced by a mixture of aromatic hydrocarbons, aliphatic olefins, and alkanes. In the hydrogenation process by using the home-made Raney $\mathrm{Ni}$ as the catalyst, the hydrogenation of the aliphatic olefins was the one of the first step to take place (Escola et al., 2012). The aromatic hydrocarbons were thereafter hydrogenated into cycloalkanes or hydroaromatic hydrocarbons via hydro-cycloaddition reactions under the very mild reaction condition. In addition, the hydroisomerization reaction could took place between the dimethylcyclohexanes; and trace volume of small hydrocarbons was also produced by hydrocracking reactions. From the 
detailed analysis of hydrogenated organics, it was found that these hydrogenated organics can be potentially used as additives to make the desired jet fuels by blending with other hydrocarbons.

\section{Conclusions}

In summary, the co-feed microwave-induced pyrolysis followed by hydrogenation process is a profound approach for the improved carbon yield of renewable alkanes for jet fuels from cellulose and LDPE. There was a positive synergy between cellulose and LDPE in the co-feed catalytic microwave pyrolysis, which could significantly improve the carbon yield of raw organics and suppress the formations of char and coke. In the hydrogenation process, four species of raw organics were hydrogenated under a low-severity condition. For the hydrogenated organics, up to $90 \%$ selectivity toward saturated hydrocarbons lumped in the jet fuel range was garnered.

\section{ACKNOWLEDGMENTS}

This study was supported partially by the Agriculture and Food Research Initiative of National Institute of Food and Agriculture, United States Department of Agriculture (Award Number: 2015-67021-22911; Award Number: 2016-67021-24533), WSU Office of Commercialization and the Offices of the President and Provost in conjunction with the Vice President of Research, the Washington Research foundation, and Chinese Scholarship Council. We are grateful to Dr. Aftab Ahamed and Dr. Xin Li for their assistance with GCMS measurements. 


\section{References}

1. Artetxe, M., Lopez, G., Amutio, M., Elordi, G., Bilbao, J., Olazar, M. 2012. Light olefins from HDPE cracking in a two-step thermal and catalytic process. Chem. Eng. J. 207-208, 27-34.

2. Bi, P., Wang, J., Zhang, Y., Jiang, P., Wu, X., Liu, J., Xue, H., Wang, T., Li, Q. 2015. From lignin to cycloparaffins and aromatics: Directional synthesis of jet and diesel fuel range biofuels using biomass. Bioresour. Technol. 183, 10-7.

3. Cheng, H., Hu, Y. 2010. Municipal solid waste (MSW) as a renewable source of energy: current and future practices in China. Bioresour. Technol. 101, 3816-24.

4. Cheng, Y.-T., Huber, G.W. 2012. Production of targeted aromatics by using Diels-Alder classes of reactions with furans and olefins over ZSM-5. Green Chem. 14, 3114.

5. Dorado, C., Mullen, C.A., Boateng, A.A. 2014. H-ZSM5 Catalyzed Co-Pyrolysis of Biomass and Plastics. ACS Sustainable Chem. Eng. 2, 301-311.

6. Dorado, C., Mullen, C.A., Boateng, A.A. 2015. Origin of carbon in aromatic and olefin products derived from HZSM-5 catalyzed co-pyrolysis of cellulose and plastics via isotopic labeling. Appl. Catal., B 162, 338-345.

7. Escola, J.M., Aguado, J., Serrano, D.P., Briones, L., Díaz de Tuesta, J.L., Calvo, R., Fernandez, E. 2012. Conversion of Polyethylene into Transportation Fuels by the Combination of Thermal Cracking and Catalytic Hydroreforming over Ni-Supported Hierarchical Beta Zeolite. Energy Fuels 26, 3187-3195.

8. Fang, S., Yu, Z., Lin, Y., Lin, Y., Fan, Y., Liao, Y., Ma, X. 2016. Effects of additives on the co-pyrolysis of municipal solid waste and paper sludge by using thermogravimetric analysis. Bioresour. Technol. 209, 265-272.

9. Green, S.K., Patet, R.E., Nikbin, N., Williams, C.L., Chang, C.-C., Yu, J., Gorte, R.J., Caratzoulas, S., Fan, W., Vlachos, D.G., Dauenhauer, P.J. 2016. Diels-Alder cycloaddition of 2-methylfuran and ethylene for renewable toluene. Appl. Catal., B 180, 487-496.

10. Gross, B.H., Mebane*, R.C., Armstrong, D.L. 2001. Transfer hydrogenolysis of aromatic alcohols using Raney catalysts and 2-propanol. Appl. Catal., A 219, 281- 289.

11. Huber, G.W., Iborra, S., Corma, A. 2006. Synthesis of Transportation Fuels from Biomass: Chemistry, Catalysts, and Engineering. Chem. Rev. 106, 4044-4098.

12. Keil, D.E., Warren, D.A., Jenny, M.J., EuDaly, J.G., Smythe, J., Peden-Adams, M.M. 2003. Immunological function in mice exposed to JP-8 jet fuel in utero. Toxicol. Sci. 76, 347-56.

13. Lemoine, F., Maupin, I., Lemee, L., Lavoie, J.M., Lemberton, J.L., Pouilloux, Y., Pinard, L. 2013. Alternative fuel production by catalytic hydroliquefaction of solid municipal wastes, primary sludges and microalgae. Bioresour. Technol. 142, 1-8.

14. Li, G., Li, N., Wang, X., Sheng, X., Li, S., Wang, A., Cong, Y., Wang, X., Zhang, T. 2014a. Synthesis of Diesel or Jet Fuel Range Cycloalkanes with 2-Methylfuran and Cyclopentanone from Lignocellulose. Energy Fuels 28, 5112-5118.

15. Li, J., Yu, Y., Li, X., Wang, W., Yu, G., Deng, S., Huang, J., Wang, B., Wang, Y. 2015. Maximizing carbon efficiency of petrochemical production from catalytic co-pyrolysis of biomass and plastics using gallium-containing MFI zeolites. Appl. Catal., B 172-173, 154-164.

16. Li, X., Li, J., Zhou, G., Feng, Y., Wang, Y., Yu, G., Deng, S., Huang, J., Wang, B. 2014b. Enhancing the production of renewable petrochemicals by co-feeding of biomass with plastics in catalytic fast pyrolysis with ZSM-5 zeolites. Appl. Catal., A, 481, 173-182.

17. Li, X., Zhang, H., Li, J., Su, L., Zuo, J., Komarneni, S., Wang, Y. 2013. Improving the aromatic 
production in catalytic fast pyrolysis of cellulose by co-feeding low-density polyethylene. Appl. Catal., A 455, 114-121.

18. Lidstrom, P., Tierney, J., Wathey, B., Westman, J. 2001. Microwave assisted organic synthesis - a review. Tetrahedron 57, 9225-9283.

19. Liu, C., Wang, H., Karim, A.M., Sun, J., Wang, Y. 2014. Catalytic fast pyrolysis of lignocellulosic biomass. Chem. Soc. Rev. 43, 7594-623.

20. Luque, R., Menéndez, J.A., Arenillas, A., Cot, J. 2012. Microwave-assisted pyrolysis of biomass feedstocks: the way forward? Energy Environ. Sci., 5, 5481-5488.

21. Miskolczi, N., Bartha, L., Deák, G., Jóver, B. 2004. Thermal degradation of municipal plastic waste for production of fuel-like hydrocarbons. Polym. Degrad. Stab. 86(2), 357-366.

22. Mullen, C.A., Boateng, A.A. 2010. Catalytic pyrolysis-GC/MS of lignin from several sources. Fuel Process. Technol. 91, 1446-1458.

23. Ren, S., Lei, H., Wang, L., Bu, Q., Chen, S., Wu, J., Julson, J., Ruan, R. 2012. Biofuel production and kinetics analysis for microwave pyrolysis of Douglas fir sawdust pellet. Journal of Analytical and Applied Pyrolysis, 94, 163-169.

24. Serrano, D.P., Aguado, J., Escola, J.M., Rodríguez, J.M., San Miguel, G. 2005. An investigation into the catalytic cracking of LDPE using Py-GC/MS. J. Anal. Appl. Pyrolysis 74(1-2), 370-378.

25. Williams, C.L., Chang, C.-C., Do, P., Nikbin, N., Caratzoulas, S., Vlachos, D.G., Lobo, R.F., Fan, W., Dauenhauer, P.J. 2012. Cycloaddition of Biomass-Derived Furans for Catalytic Production of Renewablep-Xylene. ACS Catal. 2, 935-939.

26. Xue, Y., Kelkar, A., Bai, X. 2016. Catalytic co-pyrolysis of biomass and polyethylene in a tandem micropyrolyzer. Fuel, 166, 227-236.

27. Yao, W., Li, J., Feng, Y., Wang, W., Zhang, X., Chen, Q., Komarneni, S., Wang, Y. 2015. Thermally stable phosphorus and nickel modified ZSM-5 zeolites for catalytic co-pyrolysis of biomass and plastics. RSC Adv. 5, 30485-30494.

28. Zhang, H., Carlson, T.R., Xiao, R., Huber, G.W. 2012. Catalytic fast pyrolysis of wood and alcohol mixtures in a fluidized bed reactor. Green Chem. 14, 98-110.

29. Zhang, H., Nie, J., Xiao, R., Jin, B., Dong, C., Xiao, G. 2014. Catalytic Co-pyrolysis of Biomass and Different Plastics (Polyethylene, Polypropylene, and Polystyrene) To Improve Hydrocarbon Yield in a Fluidized-Bed Reactor. Energy Fuels 28, 1940-1947.

30. Zhang, H., Xiao, R., Nie, J., Jin, B., Shao, S., Xiao, G. 2015a. Catalytic pyrolysis of black-liquor lignin by co-feeding with different plastics in a fluidized bed reactor. Bioresour. Technol. 192, 68-74.

31. Zhang, X., Lei, H., Wang, L., Zhu, L., Wei, Y., Liu, Y., Yadavalli, G., Yan, D. 2015b. Renewable gasoline-range aromatics and hydrogen-enriched fuel gas from biomass via catalytic microwaveinduced pyrolysis. Green Chem.17, 4029-4036.

32. Zhang, X., Lei, H., Yadavalli, G., Zhu, L., Wei, Y., Liu, Y. 2015c. Gasoline-range hydrocarbons produced from microwave-induced pyrolysis of low-density polyethylene over ZSM-5. Fuel, 144, 33-42.

33. Zhang, X., Lei, H., Zhu, L., Wei, Y., Liu, Y., Yadavalli, G., Yan, D., Wu, J., Chen, S. 2015d. Production of renewable jet fuel range alkanes and aromatics via integrated catalytic processes of intact biomass. Fuel, 160, 375-385.

34. Zhang, X., Lei, H., Zhu, L., Wu, J., Chen, S. 2015e. From lignocellulosic biomass to renewable cycloalkanes for jet fuels. Green Chem. 17, 4736-4747. 
35. Zhang, Y., Bi, P., Wang, J., Jiang, P., Wu, X., Xue, H., Liu, J., Zhou, X., Li, Q. 2015f. Production of jet and diesel biofuels from renewable lignocellulosic biomass. Appl. Energy 150, 128-137.

Table 1 Elemental analysis of cellulose and low-density polyethylene

\begin{tabular}{lcc}
\hline Elemental analysis $(w t \%)$ & Cellulose & Low-density polyethylene \\
\hline Carbon & 43.54 & 85.71 \\
Hydrogen & 6.43 & 14.29 \\
Nitrogen & 0 & 0 \\
Oxygen $^{\text {a }}$ & 50.03 & 0 \\
\hline
\end{tabular}

${ }^{a}$ Determined by difference 
Table 2 Textural properties of well-promoted ZSM-5 and home-made Raney Ni catalysts ${ }^{\mathrm{a}}$

\begin{tabular}{lccccc} 
& $S_{\text {BET }}$ & $V_{\text {pore }}$ & $S_{\text {pore }}$ & $d_{\text {pore }}$ & $T_{\text {acidity }}$ \\
\cline { 2 - 6 } & $\left(\mathrm{m}^{2} / \mathrm{g}\right)$ & $\left(\mathrm{cm}^{3} / \mathrm{g}\right)$ & $\left(\mathrm{m}^{2} / \mathrm{g}\right)$ & $\mathrm{nm}$ & $\mathrm{mmol} \mathrm{NH}_{3} / \mathrm{g}$ \\
\hline Parent ZSM-5 & 386.9 & 0.078 & 55.3 & 5.7 & 0.20 \\
Well-promoted ZSM-5 & 396.2 & 0.097 & 74.1 & 5.2 & 0.18 \\
Ni-Al alloy & 0.35 & 0 & 0 & 0 & - \\
Home-made Raney Ni & 52.4 & 0.034 & 35.8 & 3.8 & - \\
\hline
\end{tabular}

${ }^{\text {a }} S_{\text {BET }}$ : BET surface area; $V_{\text {pore: }}$ pore volume; $S_{\text {pore: }}$ pore surface area; $d_{\text {pore: }}$ average pore size; $T_{\text {acidity: }}$ total acidity. 
Table 3 Experimental design and product yield distribution ${ }^{\mathrm{a}}$

\begin{tabular}{cllllll}
\multirow{2}{*}{ Entryb } & Catalytic & LDPE to & \multicolumn{4}{c}{ Yield (wt\%) } \\
\cline { 4 - 6 } & Temperature $\left({ }^{\circ} \mathrm{C}\right)$ & cellulose ratio & Bio-oil & Gas & Char & Coke \\
\hline 1 & 300 & 0.5 & 45.88 & 38.10 & 14.45 & 1.57 \\
2 & 300 & 1 & 50.10 & 39.09 & 9.51 & 1.30 \\
3 & 450 & 0.5 & 38.31 & 45.90 & 14.56 & 1.23 \\
4 & 450 & 1 & 46.34 & 43.57 & 9.34 & 0.75 \\
5 & 375 & 0.75 & 45.22 & 42.51 & 11.00 & 1.27 \\
6 & 375 & 0.75 & 45.16 & 42.84 & 10.78 & 1.22 \\
7 & 375 & 0.75 & 46.02 & 42.13 & 10.59 & 1.26 \\
8 & 375 & 0.75 & 45.35 & 42.34 & 11.12 & 1.19 \\
9 & 375 & 0.75 & 45.49 & 42.41 & 10.87 & 1.23 \\
10 & 375 & 0.4 & 39.12 & 43.75 & 15.54 & 1.59 \\
11 & 375 & 1.1 & 50.45 & 40.03 & 8.63 & 0.89 \\
12 & 269 & 0.75 & 48.75 & 38.71 & 11.04 & 1.50 \\
13 & 481 & 0.75 & 42.18 & 46.23 & 10.95 & 0.64 \\
14 & 375 & 1.45 & 51.45 & 40.71 & 7.03 & 0.81 \\
15 & 250 & 0.75 & 50.27 & 37.13 & 10.87 & 1.73 \\
16 & 500 & 0.75 & 40.97 & 47.58 & 10.88 & 0.57 \\
17 & 375 & 0 & 33.54 & 48.55 & 16.09 & 1.82 \\
\hline
\end{tabular}

${ }^{a}$ Reaction condition: reaction temperature $480{ }^{\circ} \mathrm{C}$; reaction time, 10 mins.

${ }^{b}$ Entry-1 to Entry-13 were conducted based on central composite design; Entry-14 and Entry-16 were added as the controls; Entry-17 is the control in the absence of LDPE. 
Table 4 Detailed carbon yield distribution and product carbon selectivity as a function of catalytic temperature $^{\mathrm{a}}$

\begin{tabular}{|c|c|c|c|c|c|}
\hline & \multicolumn{5}{|c|}{ Catalytic temperature $\left({ }^{\circ} \mathrm{C}\right)$} \\
\hline & 250 & 269 & 375 & 481 & 500 \\
\hline \multicolumn{6}{|c|}{ Overall carbon yield (C mol\%) } \\
\hline Liquid organics & 46.35 & 45.02 & 43.87 & 40.37 & 38.87 \\
\hline Char & 15.17 & 15.41 & 15.18 & 15.28 & 15.19 \\
\hline Coke & 2.53 & 2.19 & 1.80 & 0.93 & 0.83 \\
\hline $\mathrm{Gas}^{\mathrm{b}}$ & 35.95 & 37.38 & 39.15 & 43.42 & 45.11 \\
\hline \multicolumn{6}{|c|}{ Liquid carbon selectivity (C mol\%) } \\
\hline Toluene & 5.32 & 6.23 & 5.23 & 4.89 & 4.56 \\
\hline Ethylbenzene & 6.76 & 6.34 & 7.03 & 7.66 & 8.02 \\
\hline$p$-xylene $/ m$-xylene & 18.95 & 19.35 & 23.33 & 24.55 & 26.92 \\
\hline Trimethylbenzene & 15.21 & 16.02 & 15.32 & 13.23 & 13.01 \\
\hline Indane & 1.32 & 1.43 & 1.29 & 2.03 & 2.32 \\
\hline Indene & 1.54 & 1.62 & 1.78 & 1.97 & 1.89 \\
\hline Phenol & 0.95 & 0.75 & 0.65 & 0.43 & 0.19 \\
\hline$p$-cresol $/ m$-cresol & 1.98 & 1.74 & 1.02 & 0.47 & 0.18 \\
\hline Naphthalene & 5.70 & 6.01 & 6.67 & 6.99 & 6.74 \\
\hline 1-methylnaphthalene & 5.32 & 5.78 & 6.65 & 7.43 & 7.78 \\
\hline 2-methylnaphthalene & 1.25 & 1.94 & 2.02 & 1.99 & 2.34 \\
\hline Anthracene & 0.67 & 1.03 & 1.23 & 1.45 & 1.53 \\
\hline Pyrene & 0.23 & 0.12 & 0.32 & 0.34 & 0.45 \\
\hline \multicolumn{6}{|c|}{ Gaseous carbon selectivity (C mol\%) } \\
\hline Methane & 5.25 & 6.23 & 6.43 & 5.67 & 6.02 \\
\hline Carbon monoxide & 28.32 & 24.17 & 14.26 & 16.65 & 18.23 \\
\hline Carbon dioxide & 20.35 & 18.11 & 16.74 & 18.27 & 18.45 \\
\hline Ethylene & 22.74 & 26.45 & 32.77 & 38.78 & 40.11 \\
\hline Ethane & 10.18 & 12.23 & 13.50 & 11.57 & 9.26 \\
\hline Propane $^{\mathrm{b}}$ & 13.16 & 12.81 & 16.30 & 9.06 & 7.93 \\
\hline
\end{tabular}

${ }^{a}$ Reaction condition: Catalyst, $20 \mathrm{wt} \%$ with respect to feed; LDPE, $75 \mathrm{wt} \%$ with respect to cellulose; Reaction temperature, $480{ }^{\circ} \mathrm{C}$; Reaction time, $10 \mathrm{~min}$.

${ }^{\mathrm{b}}$ Determined by difference 
Table 5 Detailed carbon yield distribution and product carbon selectivity on the basis of LDPE to cellulose ratio ${ }^{\text {a }}$

\begin{tabular}{lccccc}
\hline & \multicolumn{5}{c}{ LDPE to cellulose ratio } \\
\cline { 2 - 6 } & 0 & 0.4 & 0.75 & 1.1 & 1.45 \\
\hline Overall carbon yield (C mol\%) & & & & & \\
Liquid organics & 24.53 & 36.05 & 43.87 & 44.23 & 44.88 \\
Char & 31.78 & 24.04 & 15.18 & 11.31 & 8.83 \\
Coke & 3.76 & 2.57 & 1.80 & 1.22 & 1.06 \\
Gas $^{\mathrm{b}}$ & 39.93 & 37.34 & 39.15 & 43.24 & 45.23 \\
& & & & & \\
Liquid carbon selectivity (C mol\%) & & & & & \\
Toluene & 4.34 & 4.67 & 5.23 & 5.76 & 6.61 \\
Ethylbenzene & 5.54 & 6.32 & 7.03 & 8.05 & 9.27 \\
-xylene/m-xylene & 2.24 & 21.54 & 23.33 & 21.58 & 21.47 \\
Trimethylbenzene & 17.45 & 16.34 & 15.32 & 14.36 & 13.12 \\
Indane & 1.05 & 1.33 & 1.29 & 1.37 & 1.01 \\
Indene & 2.43 & 2.15 & 1.78 & 1.24 & 0.97 \\
Phenol & 1.25 & 0.97 & 0.65 & 0.70 & 0.43 \\
-cresol/m-cresol & 2.01 & 1.45 & 1.02 & 0.32 & 0.11 \\
Naphthalene & 9.05 & 7.97 & 6.67 & 5.23 & 4.17 \\
1-methylnaphthalene & 8.43 & 7.69 & 6.65 & 4.54 & 3.65 \\
2-methylnaphthalene & 4.78 & 3.69 & 2.02 & 1.54 & 1.01 \\
Anthracene & 2.05 & 1.07 & 1.23 & 1.04 & 0.44 \\
Pyrene & 1.24 & 0.89 & 0.32 & 0.29 & 0.17 \\
& & & & & \\
Gaseous carbon selectivity (C mol\%) & & & & & \\
Methane & 8.97 & 7.23 & 6.43 & 5.67 & 5.06 \\
Carbon monoxide & 40.78 & 26.98 & 14.26 & 12.19 & 10.21 \\
Carbon dioxide & 30.87 & 24.33 & 16.74 & 13.34 & 10.76 \\
Ethylene & 6.67 & 20.67 & 32.77 & 40.48 & 48.47 \\
Ethane & 8.50 & 11.76 & 13.50 & 12.98 & 12.21 \\
Propane & 4.21 & 9.03 & 16.30 & 15.34 & 13.29 \\
\hline a & & &
\end{tabular}

${ }^{a}$ Reaction condition: Catalyst, $20 \mathrm{wt} \%$ with respect to feed; Reaction temperature, $480{ }^{\circ} \mathrm{C}$; Catalytic temperature, $375^{\circ} \mathrm{C}$; Reaction time, $10 \mathrm{~min}$.

${ }^{\mathrm{b}}$ Determined by difference 
Table 6 Products distribution and partial alkanes' carbon selectivity for hydrogenation of diverse liquid organics ${ }^{\mathrm{a}}$

\begin{tabular}{|c|c|c|c|c|}
\hline & \multicolumn{4}{|c|}{ Organics species ${ }^{\mathrm{b}}$} \\
\hline & L-1 & $\mathrm{L}-2$ & L-3 & L-4 \\
\hline \multicolumn{5}{|l|}{ Overall selectivity (\% in area) } \\
\hline Aliphatic alkanes & - & 10.71 & 1.34 & 31.45 \\
\hline Cycloalkanes & 83.69 & 78.15 & 90.87 & 57.79 \\
\hline Hydro-aromatic hydrocarbons & 8.42 & 5.28 & 5.01 & 4.01 \\
\hline Aromatic hydrocarbons & 2.74 & 4.63 & 2.03 & 5.89 \\
\hline Others & 5.15 & 1.23 & 0.75 & 0.86 \\
\hline \multicolumn{5}{|l|}{ Alkanes selectivity (C mol\%) } \\
\hline 1,4-dimethylcyclohexane & 4.35 & 4.25 & 5.37 & 3.68 \\
\hline 1,3-dimethylcyclohexane & 11.54 & 12.78 & 16.76 & 12.11 \\
\hline 1,2-dimethylcyclohexane & 3.56 & 6.09 & 8.25 & 6.54 \\
\hline Ethylcyclohexane & 2.67 & 3.13 & 3.67 & 3.25 \\
\hline Octane & - & 0.67 & 0.15 & 1.23 \\
\hline 1,2,4-trimethylcyclohexane & 18.54 & 16.89 & 14.23 & 11.98 \\
\hline Propylcyclohexane & 4.45 & 3.21 & 3.64 & 2.13 \\
\hline Nonane & - & 0.76 & 0.09 & 1.92 \\
\hline Hexahydroindan & 4.56 & 3.32 & 4.78 & 2.21 \\
\hline Decalin & 13.65 & 6.23 & 8.23 & 4.15 \\
\hline Decane & - & 0.64 & - & 1.32 \\
\hline Undecane & - & 2.33 & 0.21 & 4.41 \\
\hline Dodecane & - & 0.76 & - & 1.47 \\
\hline Tridecane & - & 0.28 & 0.12 & 1.89 \\
\hline Tetradecane & - & 0.65 & - & 2.08 \\
\hline Pentadecane & - & 0.41 & - & 2.15 \\
\hline Hexadecane & - & 0.38 & 0.11 & 1.76 \\
\hline
\end{tabular}

${ }^{a}$ Reaction condition: Initial pressure, $500 \mathrm{psi}$; Raney Ni catalyst, $20 \mathrm{wt} \%$ with respect to reactant mass; Reaction temperature, $200{ }^{\circ} \mathrm{C}$; Reaction time, $2 \mathrm{~h}$.

${ }^{b} \mathrm{~L}-1$ : reactant from the experiment conducted at catalytic temperature of $375^{\circ} \mathrm{C}$ in the absence of LDPE; L-2: reactant from the experiment conducted at catalytic temperature of $375^{\circ} \mathrm{C}$ with LDPE to cellulose ratio of 0.75 ; L-3: reactant from the experiment conducted at catalytic temperature of $500{ }^{\circ} \mathrm{C}$ with LDPE to cellulose ratio of 0.75 ; L-4: reactant from the experiment conducted at catalytic temperature of 375 with LDPE to cellulose ratio of 1.45. 


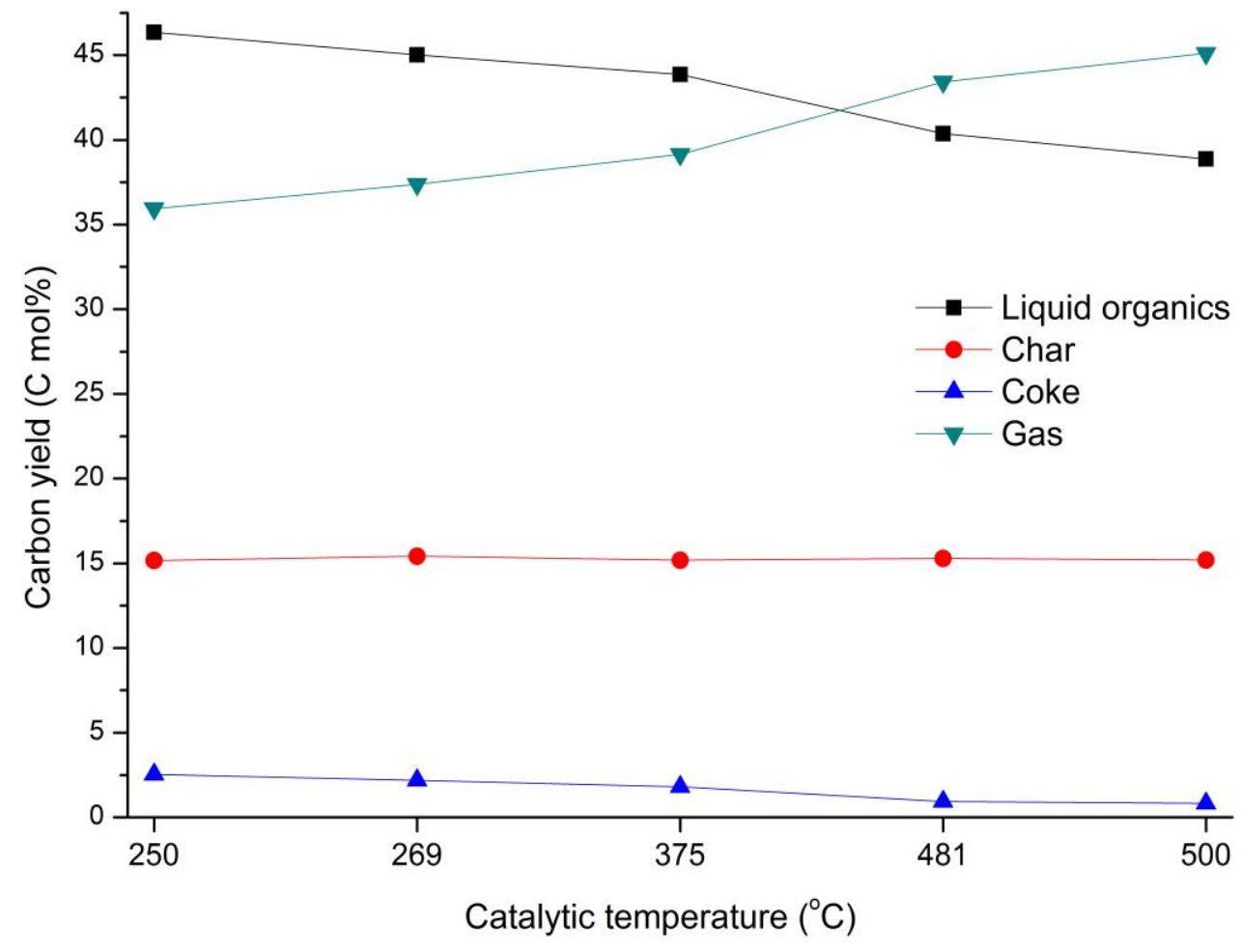

Fig. 1 The overall carbon yields regarding product distribution from co-feed catalytic microwave pyrolysis in light of catalytic temperature at the same LDPE to cellulose ratio (0.75). 


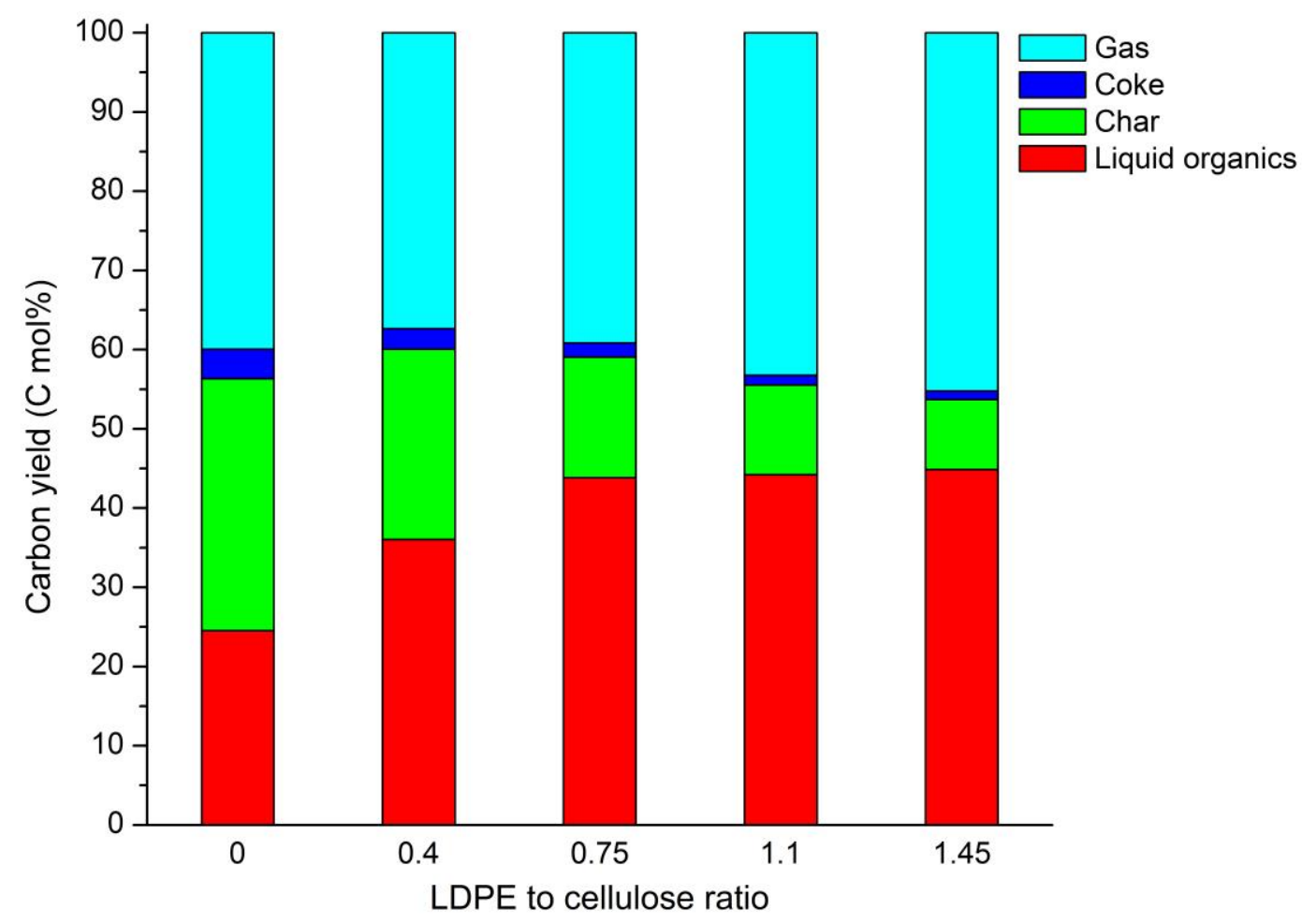

Fig. 2 The overall carbon yields regarding product distribution from co-feed catalytic microwave pyrolysis as a function of LDPE to cellulose ratio at the same catalytic temperature $\left(375^{\circ} \mathrm{C}\right)$. 


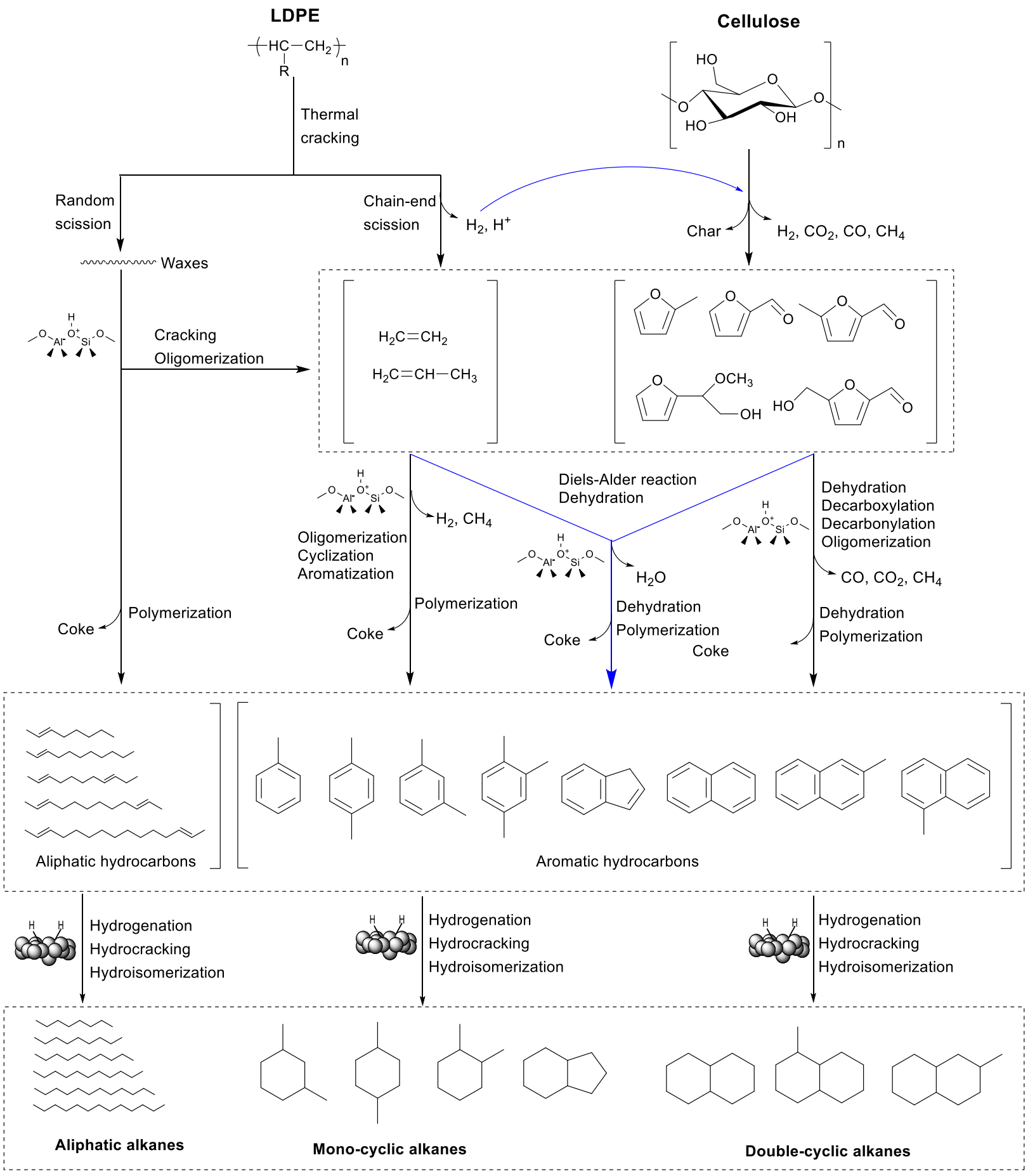

Fig. 3 Proposed reaction pathways for the conversion of cellulose and LDPE into jet fuel range alkanes. 


\section{Graphical Abstract}

Improvement of renewable alkanes for jet fuels from co-feed catalytic microwaveassisted pyrolysis and hydrogenation process
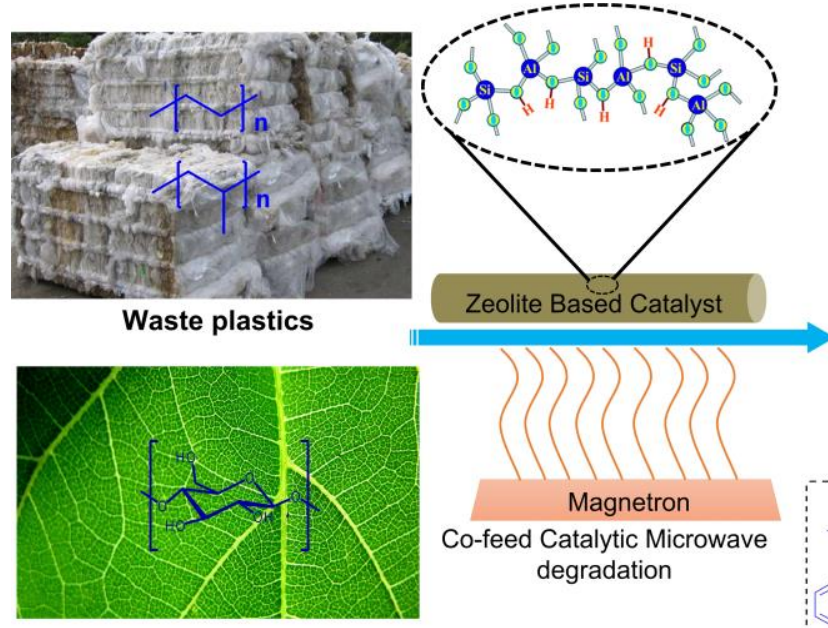

Cellulosic biomass degradation

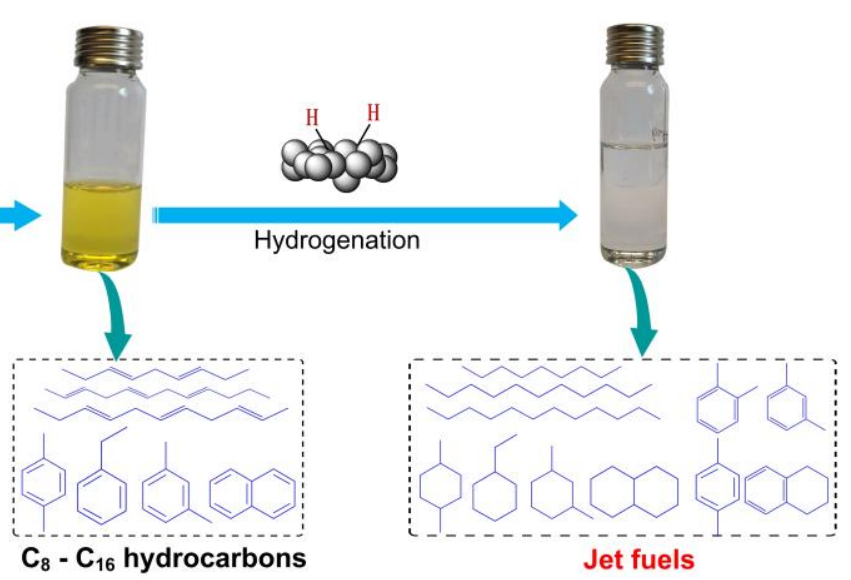

